\title{
Nouvelles données sur Coelioxys alata FöRSTER (Hymenoptera, Megachilidae), sa biologie et sa distribution
}

\author{
Par Jelle Devalez *
}

\begin{abstract}
In this article I aim to summarize all records of Coelioxys alata FÖRSTER, a rare cuckoo bee species in Europe. In addition some notes on records from Asia and the biology are provided. The habitats where the species is found are briefly discussed from all records known so far. Recently, the species was recorded at several locations in the Netherlands and Belgium and is found for the first time in France. A distribution map for Europe is created.
\end{abstract}

Résumé. Dans cet article, je fournis une synthèse des données biogéographiques relatives à Coelioxys alata FÖRSTER, est une espèce d'abeille cleptoparasite rare en Europe. Je présente également quelques notes sur les données provenant d'Asie et sur la biologie de cette espèce. Les habitats qui abritent cette espèce sont brièvement décrits sur base des données disponibles. Cette abeille a été découverte à plusieurs endroits et pour la première fois aux Pays-Bas et en Belgique, ainsi qu'en France. Une carte illustrant la distribution des observations est présentée dans cet article.

Mots-clés. Coelioxys alata, distribution géographique.

\section{Introduction}

Coelioxys alata FÖRSTER 1853 est une abeille cleptoparasite relativement rare dans la plupart des zones climatiques européennes malgré sa distribution géographique assez étendue. On retrouve également cette espèce en dehors de l'Europe, notamment en Chine, au Japon, en Russie et en Turquie (Osychnyuk et al. 1978; Enkulu 1989; Warncke 1992; Özbek \& van der Zanden 1994; Romankova 1995; Proshchalykin 2006; Nagase 2006; Wu 2006). Dans cet article, je fournis un résumé des données biogéographiques relatives à cette espèce rare en Europe, ainsi que quelques notes relatives à sa biologie.

Les premières observations liées à C. alata remontent à la fin du $19^{\text {ème }}$ siècle, lorsqu'Arnold FÖRSTER en entreprit sa description. A cette époque, les données relatives à $C$. alata étaient particulièrement peu abondantes en Europe et l'essentiel des données concernaient des individus femelles qui disposent de caractéristiques morphologiques remarquables : leur $5^{\text {ème }}$ sternite est fortement élargi en forme d'éventail et couvert de poils clairs chez les spécimens fraîchement éclos. Les mâles de $C$. alata partagent de nombreuses caractéristiques morphologiques avec les mâles de $C$. inermis et $C$. elongata; ils sont généralement d'un gabarit plus important et on les reconnaît notamment par la forme de leur capsule génitale et de leur $6^{\text {ème }}$ sternite,

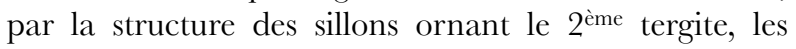
épines caractéristiques présentes sur l'apex de l'abdomen et la longueur des segments antennaires

* Adresse actuelle: BARG-EAST, BLDG 308, RM 12410300 Balt. Ave., Beltsville, MD 20705, USA. E-mail: jelle.devalez@gmail.com
(Osychnyuk et al. 1978; Warncke 1992; Banaszak \& Romasenko 1998; Amiet et al. 2004; Nagase 2006; Scheuchl 2006). En Europe, la distribution de C. elata est limitée au nord par la Finlande, à l'est par la Russie, au sud par la Bulgarie et à l'ouest par la Belgique (Figure 1).

\section{Historique des données biogéographiques}

Un siècle après sa description, seules 35 données biogéographiques fiables relatives à $C$. alata avaient été enregistrées. La connaissance de sa distribution était alors limitée à l'Allemagne (13), l'Autriche (10), la Belgique (4), les Pays-Bas (6), la République Tchèque (2), la Roumanie/Moldavie (2), la Russie (2) et la Suisse (1) (Gribodo 1884; Friese 1895, 1926; Schmiedeknecht 1930; van Lith 1937; Elfving 1968; Leclercq 1982; Peeters et al. 1999; BDFGM 2009; EIS Database 2009). Au cours des 60 dernières années, pas moins de 75 nouvelles données ont été accumulées en Allemagne (5), en Autriche (2), en Belgique (7), en Chine (1), en Estonie (6), en Finlande (2), en France (1), en Hongrie (2), au Japon (3), en Latvie (4), en Lituanie (15), aux Pays-Bas (4), en Pologne (5), en République Tchèque (5), en Russie (13), en Slovaquie (2), en Slovénie (1), et en Turquie (1) Jakubzik \& Cölln, 2007; Józan, 2006; Kowalczyk et al., 2009; Leclercq, 1982; Mandery, 2001; Monsevicius, 1995; Monsevicius, 2004; Nagase, 2006; Ostrauskas \& Monsevicius, 2002; Özbek \& van der Zanden, 1994; Pastare, 1976; Pilāts et al., 2007; Proshchalykin, 2006 \& 2009; Quest, 2009; Romankova, 1992, 1995; Tumšs, 1973; Wu, 2006). L'ensemble de ces données sont reprises dans la Figure 1. 


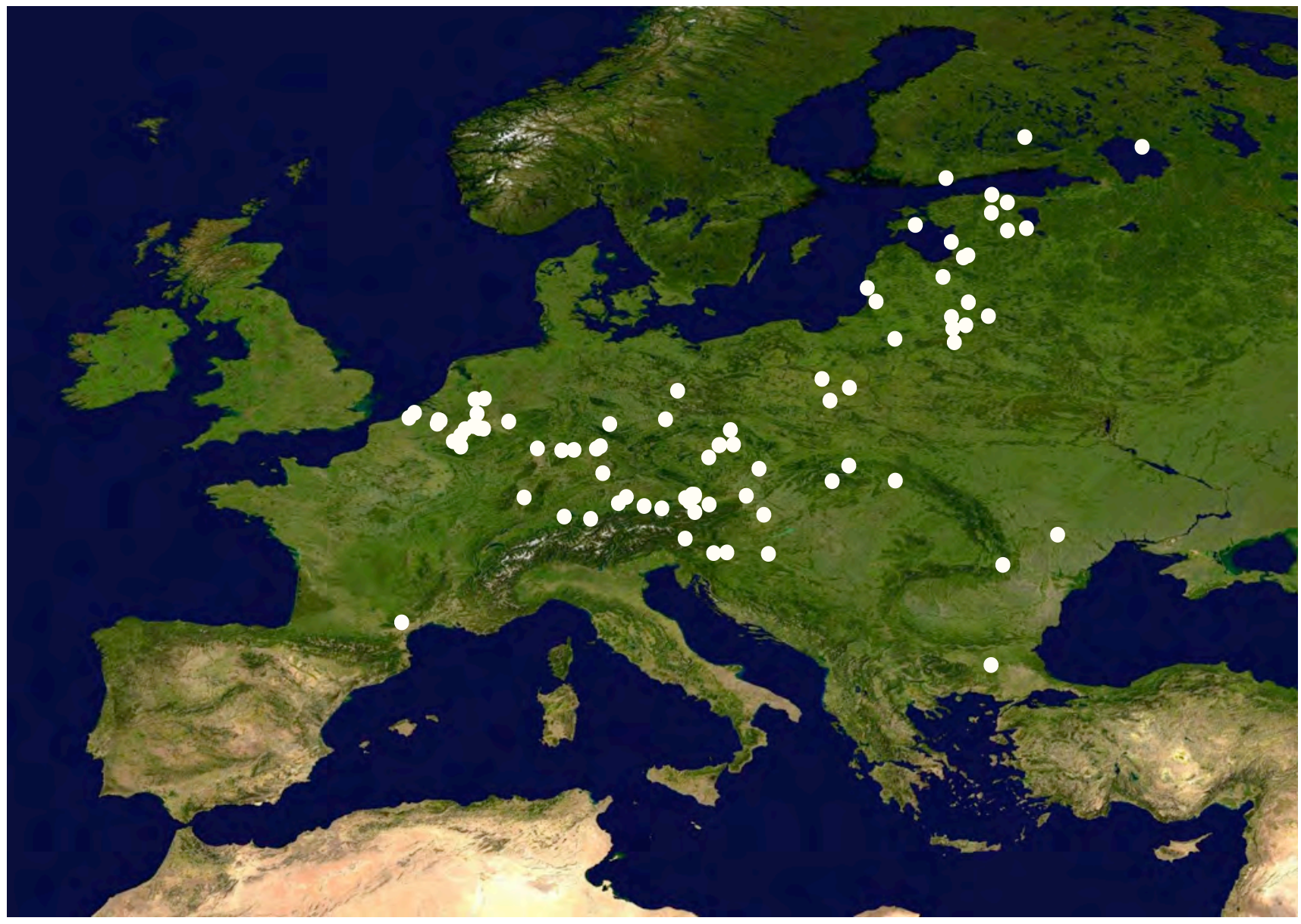

Figure 1. Distribution des données biogéographiques de Coelioxys alata FÖRSTER en Europe (illustration NJ Vereecken, fond de carte NASA). La carte illustre la présence de cette espèce dans les pays suivants: Allemagne, Autriche, Belgique, Bulgarie, Estonie, Finlande, France, Hongrie, Latvie, Lituanie, Pays-Bas,

Pologne, République Tchèque, Roumanie/Moldavie, Slovaquie, Slovénie, Suisse, est de la Turquie (non montré ici) et dans la partie européenne de la Russie.

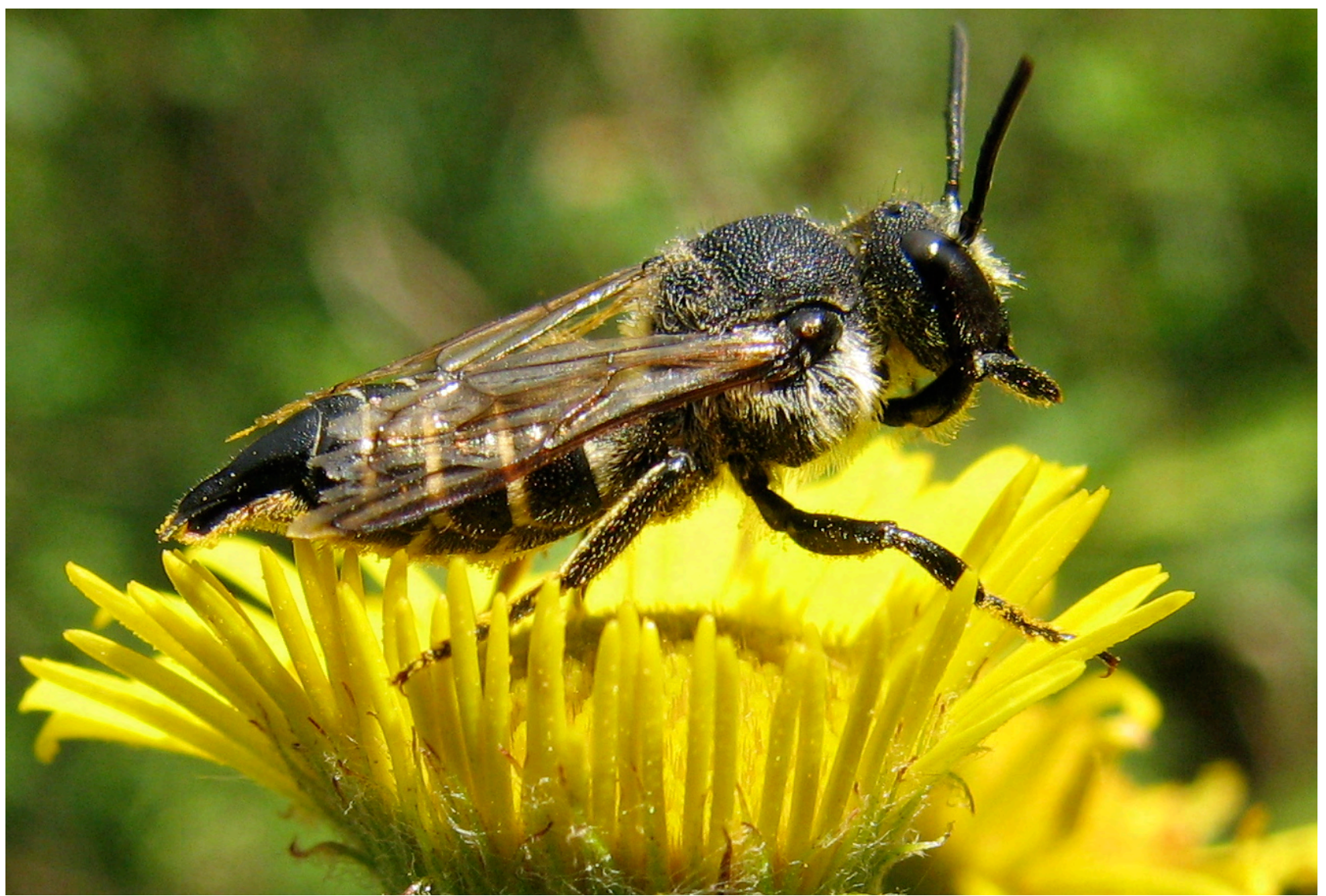

Figure 2. Femelle de Coelioxys alata FöRSTER se nourrissant sur Pulicaria dysenterica (Asteraceae) (Photo J Reyniers). 
En 2009 et 2010, j'ai non seulement eu l'occasion de récolter de nouvelles données de Belgique et des PaysBas, mais j'ai également pris connaissance de la présence de C. alata en France (H Nieuwenhuijsen 2010, comm. pers.). Les spécimens français ont été récoltés le long de la rivière "Le Jaur" à proximité de Julio sur la commune d'Olargues (département de l'Hérault). L'habitat de $C$. alata à cet endroit est similaire à celui des autres pays mais il s'agit là de la première donnée à proximité de la région méditerranéenne.

\section{Discussion}

L'abeille coucou C. alata semble caractérisée par une distribution de type amphipaléarctique. D'après les données récentes, nous pouvons également conclure que $C$. alata occupe des habitats particuliers. En effet, la plupart des sites où l'espèce a été trouvée en Belgique et aux Pays-Bas correspondent à des marais et autres milieux inondables (parfois forestiers) dans la vallée de la Meuse, l'Escaut et d'autres affluents, y compris les dunes arborées inondables. Cette abeille a été capturée dans les mêmes types de milieux partout en Europe, dans les vallées du Danube, du Rhin et leurs affluents (Isar, Iller, Main, Dráva) en Allemagne, en Autriche, et en Hongrie. En Russie, l'espèce a été détectée le long des rives de la Volga. Je dispose également d'informations sur la distribution de $C$. alata sur le pourtour de lacs continentaux dans les pays précités (Altmühlsee, Starnberger See, Chiemsee, Ossiacher See, Neusiedler See/Fertő tó et le lac Ladoga).

Globalement, il semble que les ripisylves correspondent à l'habitat-type de cette abeille coucou, notamment dans les régions d'altitude (Figure 1). Cependant, il convient de noter que C. alata a également été trouvée dans des forêts de petite taille sur sols secs. Les marais et autres milieux inondables ne sont pas réputés pour abriter une diversité remarquable d'Apoïdes. L'apparente rareté de $C$. alata pourrait s'expliquer en partie par un plus faible effort d'échantillonnage de ce type de milieu par rapport aux zones xérothermiques où la diversité d'abeilles sauvages atteint les niveaux les plus élevés (Michener 2007). Des études sur la diversité des abeilles sauvages dans les milieux a priori moins favorables pourraient déterminer si C. alata est plus fréquemment observée que ce qu'indiquent les données actuelles (Bogusch 2005).

Cette abeille cleptoparasite est estivale, sa période d'activité s'étend de juin à septembre et la presque totalité des données récoltées proviennent d'individus récoltés en juillet et en août. L'écologie de $C$. alata demeure largement inexplorée à ce jour. Comme chez la plupart des abeilles-coucou, les femelles ne semblent pas montrer de préférences florales : elles ont ainsi pu être observées récoltant du nectar sur Lythrum hyssopifolia et L. salicaria (Lythraceae), Knautia arvensis (Dipsacaceae), Melilotus albus (Fabaceae), Betonia officinalis (Lamiaceae), Rubus sp. (Rosaceae), Pulicaria dysenterica (Figure 2) et de nombreuses autres Asteraceae (Bogusch 2005; J Devalez, obs. non publiées).
Les hôtes de $C$. alata n'ont pas encore été clairement identifiés même si Megachile lapponica THOMSON 1872 et M. ligniseca KIRBY 1802 (Megachilidae) semblent être les hôtes les plus probables (Scheuchl, 2006; M. Schwarz, comm. pers. 2009). Des spécimens de C. alata n'ont jamais été découverts au sein des nids de ces dernières, mais des individus ont été trouvés dans des nids artificiels au sein et aux environs desquels $M$. ligniseca nidifiait (Villu, pers. comm. 2009). Le recouvrement phénologique important entre $M$. ligniseca et $C$. alata en de nombreux endroits semble également indiquer une interaction entre ces deux espèces, même si cela n'a pas encore été confirmé. Le même type de données indiquent que dans la région de Primorsky Krai (Russie orientale), $M$. lapponica est probablement l'hôte de $C$. alata (M. Schwarz, comm. pers. 2009). Depuis la publication de Schmiedeknecht (1930), Anthophora furcata (Apidae) a également été suggérée comme hôte mais aucune autre observation n'a confirmé cette interaction.

La plupart des espèces du genre Coelioxys déposent leurs oeufs dans les nids des Megachile. Les femelles de ces abeilles coucous ont un abdomen allongé et à l'extrémité de forme conique, un caractère qui pourrait représenter une adaptation à la ponte dans des cellules larvaires hôtes en cours d'approvisionnement (Westrich 1989; Bogusch 2005; Rozen \& Kamel 2007). Les femelles des Coelioxys ne pondent qu'un seul oeuf par cellule parasitée et à ce jour, on leur connaît deux stratégies de parasitisme :

- la femelle coucou dépose un oeuf sur l'oeuf de l'hôte qui repose lui-même sur les provisions accumulées dans la cellule larvaire. L'embryon des Coelioxys se développe généralement plus rapidement que celui de leur hôte, ce qui permet à la larve de l'abeille coucou d'émerger en premier et de tuer immédiatement la larve de l'hôte. Ce mode de parasitisme a été décrit chez C. afra LEPELETIER 1841 et chez C. coturnix PÉREZ 1884 (Rozen \& Kamel 2008);

- la femelle coucou cache son oeuf au coeur de la cellule larvaire hôte pendant que cette dernière est encore en cours d'approvisionnement et avant la ponte de l'oeuf par la femelle hôte. Une fois arrivée au troisième stade larvaire, la larve de l'abeille coucou est capable de tuer la larve hôte et se développe donc seule au sein de la cellule parasitée.

Des recherches récentes indiquent que les genres parasites Coelioxys et Radoszkowskiana au sein des Megachilini ont probablement une origine commune et que la seconde stratégie de parasitisme serait effectivement dérivée de la première chez certaines espèces au cours de l'évolution. La stratégie déployée par C. alata n'a pas encore fait l'objet d'études spécifiques, donc à ce stade nous ne pouvons que spéculer sur le mode de parasitisme chez cette espèce. L'origine de la forme allongée du 5ème sternite et son rôle dans l'oviposition demeurent inconnues. 


\section{Remerciements}

Je remercie chaleureusement toutes les personnes qui m'ont aidées à préparer cet article en fournissant des données historiques et/ou non publiées ainsi que des illustrations : A. Müller, A. Gogala, A. Grace, E. Budrys, E. De Bree, F. Kok, H. Schwenninger, H. Nieuwenhuijsen, H. Nagase, I. Raemakers, J. Luig, J. D'Haeseleer, J. Ascher, J. Reyniers, J. Pöyry, K. Cölln, L. Calle, M. Poikans, M. Proshchalykin, M. Schwarz, M. Reemer, N. Vereecken, P. Bogusch, S. Roberts, T. Pawlikowski, T. Peeters, V. Soon et Y. Barbier. Je remercie également $\mathrm{N}$. Vereecken pour la traduction de l'article et la préparation de la carte de distribution.

\section{Références bibliographiques}

Amiet F, Herrmann M, Müller A \& Neumayer R, 2004. Fauna Helvetica 9, Apidae 4: Anthidium, Chelostoma, Coelioxys, Dioxys, Heriades, Lithurgus, Megachile, Osmia, Stelis. Centre Suisse de cartographie de la faune: Neuchâtel : Switzerland.

Banaszak J \& Romasenko L, 1998. Megachilid bees of Europe (Hymenoptera. Apoidea, Megachilidae). Pedagogical University of Bydgoszcz.

BDFGM, 2009. Banque de données faunistique de Gembloux \& Mons, 2009. Mons.

Benno P, 1953. De bijen- en wespenfauna in Montferland en het aangrenzende cultuurland. Dans: dr. J.H. van Heek. De Liemers, gedenkboek Didam, pp.252-266.

Bogusch P, 2005. Distribution and biology of Coelioxys alata Hymenoptera: Apoidea, Megachilidae) in the Czech Republic and Slovakia. Klapalekiana 41: 139-143.

Galle L \& Jacobusse C, 2008. Bijen en wespen in Zeeland - Fauna

Zeelandica 4 . Het Zeeuws Landschap, Wilhelminadorp.

Database European Invertebrate Survey (EIS)-Netherlands, 2009. Leiden.

Elfving R, 1968. Die Bienen Finnlands. Fauna Fennica 21 Helsinki (Finland): Societas pro.

Enkulu L, 1989. Les Mégachiles (Hymenoptera, Apoidea) d'Europe et d'Afrique . Thèse de doctorat, Faculté des Sciences agronomiques de l'Etat, Gembloux.

Friese H, 1895. Die Bienen Europa's (Apidae europaeae), Theil I, Schmarotzerbienen, Berlin, Friedländer \& Sohn.

Friese H, 1926. Die Bienen, Wespen, Grab-und Goldwespen. Franch'sche Verlagshandlung, Stuttgart.

Gribodo G, 1884. Sopra Alcune Specie Nuove o poco conosciute di Imenotteri Antofili. Bolletino della Società Entomologica Italiana 269-274.

Jakubzik A \& Cölln K, 2007. Coelioxys alata FÖRSTER, 1853, ein Wiederfund auf dem Territorium von Nordrhein-Westfalen nach über 100 Jahren. BembiX 25: 14-16.

Józan Z, 2006. Adatok Dél-Dunántúl fullánkos hártyásszárnyú (Hymenoptera, Aculeata) faunájának ismeretéhez. Natura Somogyiensis 9: 279-288.

Kowalczyk JK, Kurzac T \& Pawlikowski T, 2009. The state of knowledge on the bees (Hymenoptera, Apoidea, Apiformes) of the Wzniesienia Łódzkie Landscape Park.

Leclercq J, 1982. Hymenoptera Apoidea Colletidae, Halictidae, Melittidae, Megachilidae et Anthophoridae (partim). Dans Leclercq J, Gaspar C \& Verstraeten C (eds.) Atlas provisoire des Insectes de Belgique (et des régions limitrophes), Faculté des Sciences agronomiques de l'Etat, Gembloux, Belgique, cartes 1483-1571

Mandery K, 2001. Die Bienen und Wespen Frankens. Bund Naturschutz Forschung 5

Michener CD, 2007. The Bees of the World, second edition. The Johns Hopkins University Press, Baltimore.

Monsevičius V, 1995. A check-list of wild bee species (Hymenoptera, Apoidea) of Lithuania with data to their distribution and bionomics. Dans Jonaitis V (ed.) New and Rare for Lithuania Insect Species. Records and Descriptions of 1994-1995. Vilnius, Institute of Ecology Publishers, pp. 7-144.

Monsevičius V 2004. Comparison of three methods of sampling wild bees (Hymenoptera, Apoidea) in Èepkeliai Nature Reserve South Lithuania). Ekologija 4: 32-39.

Nagase H, 2006. Synopsis of the bee genus Coelioxys LATREILLE (Hymenoptera: Megachilidae) of Japan, with description of a new species. Entomological Science 9(2): 223-238.
Ostrauskas H \& Monsevicius V 2002. Wild bees (Apoidea, Hymenoptera) caught with green funnel traps in Lithuania. Acta Zoologica Lithuanica 12(2): 191-196.

Osychnyuk AZ, Panfilov DV \& Ponomareva AA, 1978. Famille des Megachilidae. Dans Medvedeva GS (ed.) Opredelitel' nasekomykh evropeyskoy chasti S.S.S.R. III. Pereponchatokrylye. Akademii Nauk S.S.S.R., Leningrad, pp.418-452. Traduction en français par A. Descamps, Université de Mons-Hainaut.

Özbek H \& van der Zanden G, 1994. A preliminary review of the Megachilidae of Turkey, Part V (Hymenoptera: Apoidea). Türk Entomol. Derg, 17(4): 193-207.

Pastare S, 1976. Bišu fauna Gaujas senlejā pie Siguldas. Latvijas Entomologs 18: 27-38.

Peeters TMJ, Raemakers IP \& Smit J 1999. Voorlopige atlas van de Nederlandse bijen (Apidae), EIS-Nederland, Leiden.

Pilāts V (ed.) 2007. Biodiversity in Gauja National Park, Gauja National Park Administration, Sigulda, Latvia.

Proshchalykin MYu, 2006. Bees of the genus Coelioxys LATREILLE, 1809 (Hymenoptera, Apoidea, Megachilidae) from the Russian Far East. Euroasian Entomological fournal 5(4): 318-324.

Proshchalykin MYu \& Quest M, 2009. Section Apiformes Bees. Dans Storozhenko SYu, Sundukov YuN, Lelej AS, Sidorenko VS, Proshchalykin MYu \& Kupianskaya AN (eds.). Insects of Lazovsky Nature Reserve. Dalnauka, Vladivostok, pp.238-250 (en Russe).

Quest M, 2009. Artbestand, Ökologie und Habititatwahl von Bienen ausgewählter Offenlebensräume im Lazovski Zapovednik (Ferner Osten Russland) (Hymenoptera, Apiformes). Entomofauna 15 (Suppl.), 1-357.

Ritsema C, 1882. [Hymenoptera verzameld op excursie MeerssenValkenburg] Verslag 36e zomervergadering NEV. Tijdschrift voor Entomologie 25: 15-16.

Romankova TG, 1992. Bdzholini rodini Megachilidae Zabaikal'ya Les abeilles de la famille des Megachilidae dans le Transbaikalia]. Dans Dolin VG (ed.). The 4th Congress of the Ukrainian Entomological Society (Kharkov, September 1992), Kharkov (Ukrainian Entomological Society): pp. 145-146 (en Ukrainien).

Romankova TG, 1995. Megachilidae. Dans Lehr PA (ed.). Key to the Insects of Far Eastern Russia In Six Volumes, Vol. IV, Neuropteroidea, Mecoptera, Hymenoptera, Part 1. Nauka, St. Petersburg, pp. 530-547.

Rozen JGJr \& Kamel SM, 2006. Interspecific variation in immature larvae of the cleptoparasitic bee genus Coelioxys (Hymenoptera: Megachilidae). Journal of the Kansas Entomological Society 79(4): 348-358.

Rozen JGJr \& Kamel SM, 2007. Investigations on the biologies and immature stages of the cleptoparasitic bee genera Radoszkowskiana and Coelioxys and their Megachile hosts (Hymenoptera: Apoidea: Megachilidae: Megachilini), American Museum Novitates 3573:1-43.

Rozen JGJr \& Kamel SM, 2008. Hospicidal behavior of the cleptoparasitic bee Coelioxys (Allocoelioxys) coturnix, including descriptions of its larval instars (Hymenoptera: Megachilidae), American Museum Novitates 3636:1-15.

Scheuchl E, 2006. Illustrierte Bestimmungstabellen der Wildbienen Deutschlands und Osterreichs: Band 2, 2nd expanded and revised edition.

Schmiedeknecht O, 1907. Die Hymenopteren Mitteleuropas. Verlag G. Fischer, Jena,.

Schmiedeknecht O, 1930. Die Hymenopteren Nord- und Mitteleuropas. Verlag G. Fischer, Jena.

Tumšs V, 1973. Materiāli Latvijas bišu (Hymenoptera, Apoidea) faunai. II. Zooloăijas Muzeja Raksti 11: 5-33.

van Lith JP, 1937. Natuurhistorisch Maandblad - NHM 26(9): 103-104. (waarnemingen van H.G.M. Teunissen).

Westrich P, 1989. Die Wildbienen Baden-Württembergs. Ulmer Verlag Stuttgart, Band I und II.

Westrich $\mathbf{P} \&$ Schwenninger HR, 1993. Bemerkungen zur Bienenfauna Südwestdeutschlands (Hymenoptera, Apoidea). Mitt. Ent. Ver. Stuttgart 27: 109-117.

Warncke K, 1992. Die westpaläarktischen Arten der Bienengattung Coelioxys LATR. (Hymenoptera, Apidae, Megachilidae). Bericht der Naturf. Gesellsch. Augsburg 196: 31-77.

Wu Y, 2006. Fauna Sinica: Insecta Volume 44: Hymenoptera Megachilidae. Beijing.

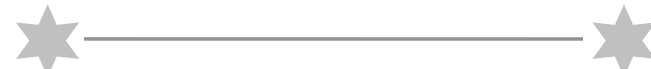

